\title{
TRIgeminal NeURALGiA CAUSEd by INTRACRANIAL EPIDERMOID TUMOR: REPORT OF A CASE AND REVIEW OF THE Different Therapeutic Modalities
} \begin{abstract}
Bayram Cirak, MD, Nejmi Kiymaz, MD
Trigeminal neuralgia (TGN) is char-
acterized by recurrent paroxysms of unilateral facial pain that typically is severe, lancinating, and activated with cutaneous stimulation. Paroxysms typically last for 1 to 2 seconds. Etiology includes compression of the trigeminal nerve by vascular structures, tumors and multiple sclerosis plaques in the medulla spinalis. TGN is rather rare as a presenting symptom with intracranial tumors. Epidermoid tumors comprise $1 \%$ of all intracranial tumors. The
\end{abstract}

Trigeminal neuralgia (TGN), or tic dolourex, is a debilitating syndrome consisting of unilateral short bursts of lancinating pain in the distribution of one or more branches of the trigeminal nerve. Typically, touching specific trigger points, speaking, or eating can provoke pain. The incidence of TGN is reported to be 4.3 per 100,000 people and occurs predominately in females. Pathogenesis includes vascular compression of trigeminal nerve roots, intracranial tumors, intracranial infections and MS plaques in the medulla spinalis (1).

Any intracranial tumor causing 5th nerve compromise anywhere along its course may cause TGN. PCA epidermoid tumors, although of benign nature, are of considerable neurosurgical interest because of their close proximity and adherence to the cranial nerves and brain stem. Epidermoid tumors comprise $1 \%$ of all intracranial tumors. They arise from dorsal midline ectodermal remnant cells. In the

From Department of Neurosurgery and Department of Neuroradiology, Johns Hopkins Medical Institutions, Baltimore, MD, and Department of Neurosurgery, School of Medicine, Yuzuncu Yil University, Van, Turkey. Address Correspondence: Bayaram Cirak, MD, 103 Willow Bend Dr. 4-D, Owings Mills, MD 21117.

Email: bayramcirak@yahoo.com

Funding: There was no funding in preparation of this manuscript.

Conflict of Interest: None majority of epidermoid tumors are located at the pontocerebellar angle, the third ventricle, and the suprasellar region. Symptomatology is not different from other tumors located at the same sites. Trigeminal neuralgia may be caused by tumor compressing the nerve itself, an inflammatory response to the epidermoid tumor or vascular compression of the nerve.

We describe the case of a 30-yearold female presenting with right-sided facial pain. Her past history revealed mul- tiple teeth extractions done to relieve facial pain. Radiological evaluation, including magnetic resonance imaging (MRI), showed a pontocerebellar angle (PCA) epidermoid tumor. TGN resolved completely after total tumor resection. A temporary facial paralysis consequent to surgical removal of the tumor improved 6 months postoperatively, and she remained painfree, without medications.

Keywords: Brain tumor, epidermoid tumor, trigeminal neuralgia. majority of cases, they are located at the PCA. Histopathologically, epidermoid tumors have one-layered stratified squamous epithelia. They are benign tumors; malignant transformation is rather rare (2-4).

\section{CASE Report}

A 30 year-old woman presented with the chief complaint of right facial pain of 8 years' duration. The pain was sharp, intermittant on the right cheek and provoked by eating and speaking. Evaluation of her past history revealed multiple tooth extractions of the molar and premolar teeth bilaterally, based on the erroneous belief that pain was dental in orgin. On neurologic examination, cranial nerve functions were normal. She had neither cerebellar nor brainstem signs. Her chewing, swallowing, hearing, facial motor and sensory functions were normal. The uvula was in the midline and the gag reflex was intact.

Since her complaint continued after teeth extractions, she was referred to

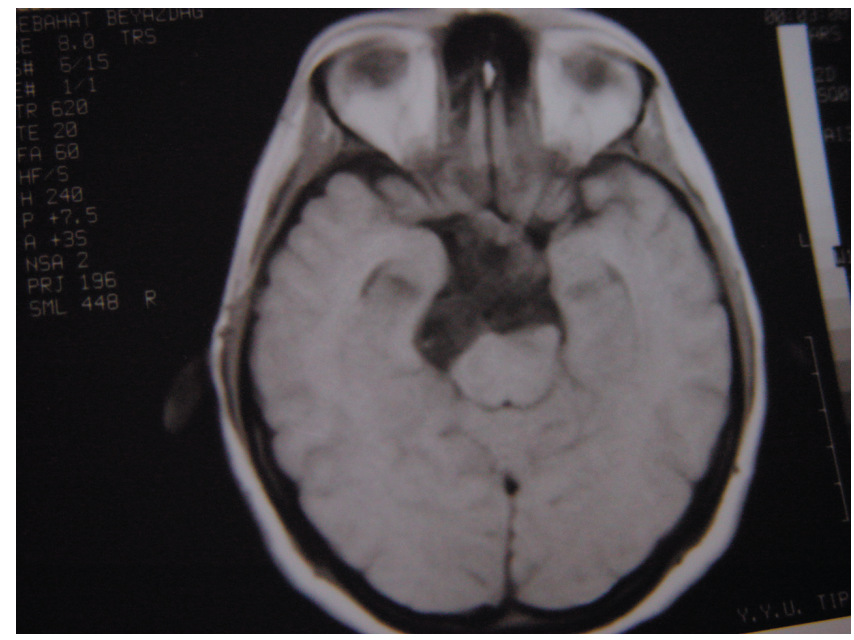

Fig 1. Cross section view of T1-weighted image of tumor. 


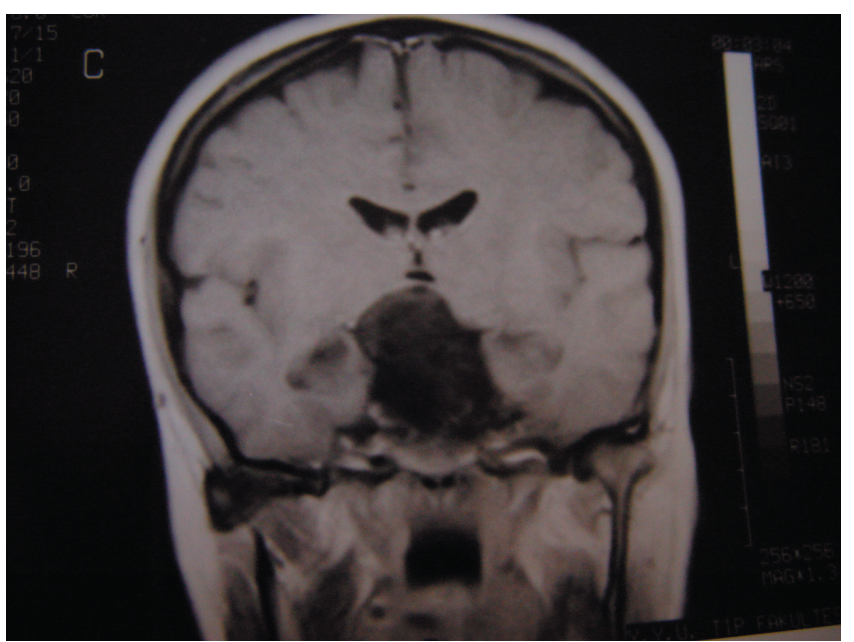

Fig 2. Coronal T1-weighted image of the tumor.

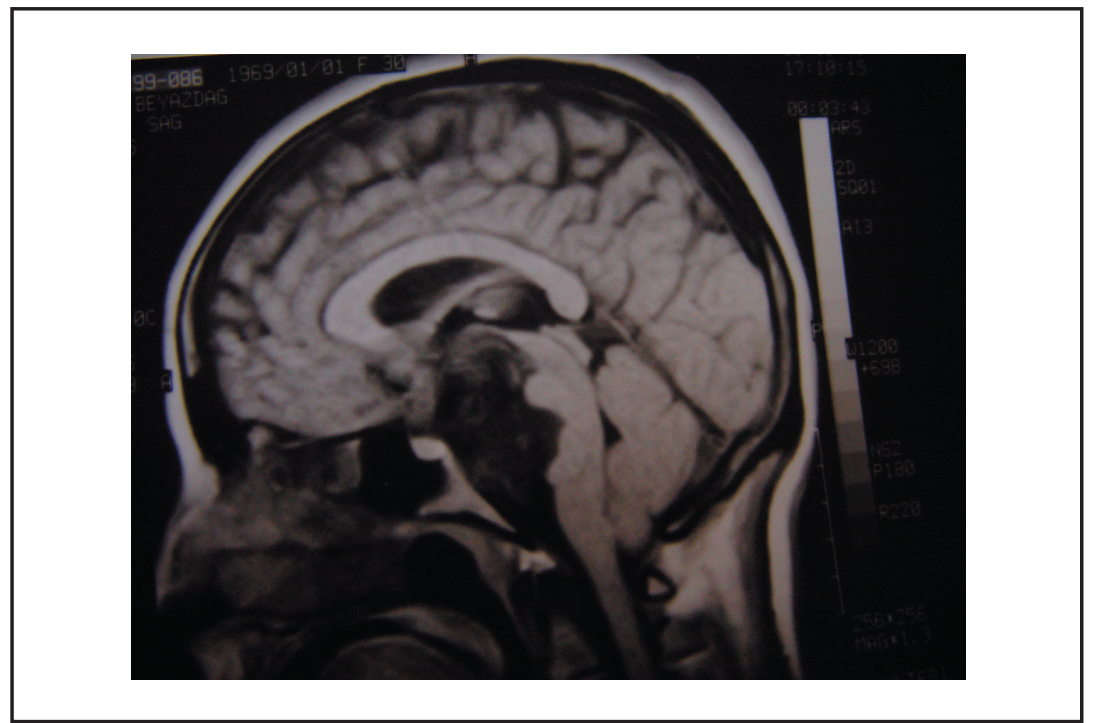

Fig 3. T1-weighted sagittal section of the tumor compressing the brainstem.

a neurologist and put on carbamazepine treatment with the diagnosis of "trigeminal neuralgia". The medication did not help, so a few weeks later, baclofen was added to the treatment. Subsequently, she consulted the neurosurgery clinic, with the diagnosis of "medically resistant trigeminal neuralgia", to be evaluated for a surgical intervention. At that time, she underwent a cranial MRI, which demonstrated an intracranial tumor anterior to the brainstem. The tumor was hypointense on T1-weighted images, and distorted the brainstem. The tumor was located along the course of the trigeminal, facial and vestibulocochlear nerves on the

\section{DISCUSSION}

Trigeminal neuralgia, although a common diagnosis, only rarely is due to intracranial tumors (incidence $<0.8 \%$ ) (3). Tumors causing trigeminal neuralgia are generally located in the posterior fossa and are usually acoustic neuromas. Tumors located at the central fossa tend to be meningiomas, schwannomas and pituitary adenomas. Epidermoid tumors as a cause of TGN are rather rare tumors and constitute only $1 \%$ of all intracranial tumors $(5,6)$.

Epidermoid tumors generally are located at the pontocerebellar angle, but may occur in the fourth ventricle and suprasellar region. Epidermoid tumors are covered with stratified squamous epithelium and contain keratin (desquamous epitel), cell debris and cholesterol. Intracranial epidermoid tumors are slow-growing congenital neoplasms that usually spread and adhere to the critical neurovascular structures along the basal cistern, particularly the PCA and parasellar region. Clinical symptoms include trigeminal neuralgia, headache and dizziness, progressive hemiparesia, unstable gait and hemifacial spasm. Epidermoid tumors surround vital structures like cranial nerves, brainstem and vascular structures.

On MRI, T1-weighted images have a higher intensity than cerebrospinal fluid (CSF); T2-weighted images are isointense with CSF. Treatment is total surgical resection of the tumor. Tumors of the PCA and posterior fossa may present with only cranial nerve dysfunction. Epidermoid tumors are atypical, because they do not infiltrate adjacent normal neural structures and may grow to a huge extent before any signs develop. Because of this, in cases of facial pain resembling TGN, a detailed radiological examination, preferably MRI, including evaluation of the brain and brainstem, is warranted (7-12)

How vascular compression of the trigeminal nerve provokes trigeminal neuralgia is still not clear. Focal demyelination caused by chronic compression and cross excitation between adjacent touch and pain-conducting fibers is the proposed mechanism (13). Although the pathogenesis of TGN is not clear, various medical and invasive therapeutic procedures have been described over the years. The initial treatment is medical: carbamazepine is the drug of choice. Baclofen, or a combination of drugs, such as carbam- 
azepine and baclofen, can also be used. Most patients initially respond to medical treatment, but $30-75 \%$ do not obtain long-term pain relief or experience side effects of the drugs (14). For these patients, surgical intervention should be considered.

There is a continuing struggle between supporters of percutaneous ablation of the ganglion of Gasser and advocates of microvascular decompression of the trigeminal nerve root $(15,16)$. The idea that TGN might be caused by vascular compression is based on Dandy's observations in the 1930s. If no vascular compression is found at operation, the sensory root can be partially transected as described by Dandy in 1934. The surgical technique for microvascular decompression is simply to remove the offending vessel and insert a piece of Teflon as popularized by Jannetta in 1985 .

The most widely used percutaneous procedure is percutaneous controlled differential thermocoagulation, as outlined by Sweet and Wepsic in 1974. It is an ambulatory procedure, requiring only brief anesthesia. A needle is placed transcutaneously through the foramen ovale into the Gasserian ganglion under direct Xray control. Once the position of the needle has been verified in the conscious patient by electrical stimulation, the affected trigeminal division is coagulated. Altering the electrical current and duration of $\mathrm{co}^{-}$ agulation controls the amount of destruction. The aim of this procedure is to destroy unmyelinated and small myelinated pain conducting fibers while sparing the thick myelinated touch conducting fibers, thus preserving touch sensation.

Jannetta described microvascular decompression as sparing the nerve and causing a high proportion of immediate relief $(90 \%)$, and those patients who are free of pain one year after the operation will suffer no recurrence (16). He also states that if the patient had a prior destructive procedure, decompression would be less effective. Both procedures achieve a high proportion of immediate relief in vascular compressive trigeminal neuralgia. The surgical results, in tumor cases, were excellent in patients with near-total tumor removal, as well as in patients with total removal. Aggressive surgical removal results in transient but significant cranial nerve dysfunction in the postoperative period. A conservative approach is indicated for patients in whom the capsule is adherent to the brain stem and the cranial nerves. Perioperative administration of steroids and wound protection may be beneficial for preventing the development of postoperative aseptic meningitis and hydrocephalus (17).

Trigeminal nerve neuropathy may involve its full course, from nuclei in the brain stem to peripheral branches. The nerve can be divided into four segments for simplifying the differential diagnoses: extracranial, cavernous sinus, Meckel's cave and brain stem. Multiple sclerosis, infarct, and glioma are the most common abnormalities in the brain stem leading to trigeminal neuropathy. Neurovascular compression, followed by acoustic and trigeminal schwannomas, meningiomas, epidermoid cysts, lipomas, and metastases are the most common cisternal causes. Trigeminal neuropathy arising from Meckel's cave and cavernous sinus is frequently due to meningiomas, trigeminal schwannomas, epidermoid cysts, metastases, pituitary adenomas, and aneurysms. The most common extracranial causes are malignant tumors, which may demonstrate perineural tumor spread. Because the clinical findings may not help in differential diagnosis as in our case, MRI must be used to visualize the entire course of the fifth cranial nerve. The standard study should include T2-weighted images of the whole brain and highresolution axial and coronal T1-weighted images of the skull base obtained with and without contrast material enhancement $(3,5,8)$.

TGN is a syndrome caused by a variety of mechanisms, including vascular compression, intracranial infections, demyelinating diseases and intracranial tumors. Epidermoid tumor as a cause of TGN is very rare. In those cases with epidermoid tumor, trigeminal neuralgia can be caused either by tumor compression of the nerve or an inflammatory reaction to the tumor. Anatomical changes in the route of the vascular structures caused by tumor compression may also play a role in the development of trigeminal neuralgia. Microvascular decompression may be needed in addition to tumor removal in such cases.

In summary, we describe a case of intractable trigeminal neuralgia that resulted from a large, benign, brainstem epidermoid tumor. This presentation underscores the fact that imaging of intracranial structures, including the brainstem and posterior fossa, is warranted. MRI is the radiologic study of choice. The patient was pain free after surgical excision of the tumor. The case provides an opportunity to review the pathogenesis, diagnosis and treatment of trigeminal neuralgia.

\section{Author Affiliation: \\ Bayram Cirak, MD \\ 103 Willow Bend Dr. 4-D \\ Owings Mills, MD 21117 \\ Email: bayramcirak@yahoo.com \\ Nejmi Kiymaz, MD \\ Department of Neurosurgery \\ School of Medicine \\ Yuzuncu Yil University \\ Van, Turkey \\ Atilla Arslanoglu MD \\ Department of Neuroradiology \\ Johns Hopkins Medical Institutions \\ 600 Caroline Suite \# 50738 \\ Baltimore, MD 21287}

\section{RefEREnCES}

1. Katusic S, Beard CM, Bergstralh Eet al. Incidence and clinical features of trigeminal neuralgia, Rochester, Minnesota, 19451984. Ann Neurol 1990; 27:89-95.

2. Fujita N, Hirabuki N, Kashiwagi Net al. Epidermoid tumor of the cerebellopontine angle: signal loss in the contralateral cistern. AJNR Am J Neuroradiol 1998; 19:333-335.

3. Desai K, Nadkarni T, Bhayani R et al. Cerebellopontine angle epidermoid tumor presenting with 'tic convulsif' and tinnitus--case report. Neurol Med Chir (Tokyo) 2002; 42:162-165.

4. Mohanty A, Venkatrama SK et al. Experience with cerebellopontine angle epidermoids. Neurosurgery 1997; 40:24-29.

5. Rappaport ZH. Epidermoid tumour of the cerebellopontine angle as a cause of trigeminal neuralgia. Neurochirurgia (Stuttg) 1985; 28:211-212.

6. Iwasaki K, Kondo A, Otsuka S et al. Painful tic convulsif caused by a brain tumor: case report and review of the literature. Neurosurgery 1992; 30:916-919.

7. Kapila A, Steinbaum S, Chakeres DW. Meckel's cave epidermoid with trigeminal neuralgia: CT findings. J Comput Assist Tomogr 1984; 8:1172-1174.

8. Shinoda S, Kusama R, Chou H et al. A case of painful tic convulsif due to cerebellopontine angle epidermoid tumor which could not be clearly detected by MRI. No Shinkei Geka 1995; 23:599-602.

9. Majoie CB, Verbeeten B, Dol JA et al. Trigeminal neuropathy: Evaluation with MR imaging. Radiographics 1995; 15:795-811.

10. Fitt AW, Pigott TJ, Marks PV. Undiagnosed epidermoid cyst presenting as trigeminal neuralgia: a need for MRI. Br J Neurosurg 1994; 8:101-103.

11. Tekkok IH, Suzer T, Erbengi A. Non-acous- 
tic tumors of the cerebellopontine angle. Neurosurg Rev 1992; 15:117-123.

12. Ogleznev KYa, Grigoryan YuA, Slavin KV. Parapontine epidermoid tumours presenting as trigeminal neuralgias: anatomical findings and operative results. Acta Neurochir (Wien) 1991; 110:116-119.

13. Dandy WE. Concerning the cause of trigeminal neuralgia. Am / Surg 1934; 24:
447-455

14. Taylor JC, Brauer S, Espir ML. Long-term treatment of trigeminal neuralgia with carbamazepine. Postgrad Med / 1981; 57:16-18.

15. Sweet WH, Wepsic JG. Controlled thermocoagulation of trigeminal ganglion and results for differential destruction of pain fibers. Part 1: Trigeminal neuralgia. J Neuro- $\operatorname{surg} 1974 ;$ 40:143-156.

16. Jannetta PJ. Microsurgical management of trigeminal neuralgia. Arch Neurol 1985 42; 800 .

17. Otsuka S, Nakatsu S, Matsumoto S et al. Epidermoid tumor presenting with trigeminal neuralgia and ipsilateral hemifacial spasm: a case report. Nippon Geka Hokan 1989; 58:245-249. 